\title{
PIECEWISE UNIFORM PRODUCT POLAR QUANTIZATION
}

\author{
Zoran H. Peric, Daniela M. Milovic \\ Faculty of Electronic Engineering, \\ University of Nis, Serbia \& Montenegro \\ e-mail peric@elfak.ni.ac.yu
}

\begin{abstract}
In this paper, simple and complete asymptotical analysis is given for a piecewise uniform product polar quantizer (PUPPQ) with respect to mean-square error (MSE) i.e. distortion (D). PUPPQ is based on uniform product polar quantizers. PUPPQ consists of L different uniform product polar quantizers. Uniform product polar quantizer conditions for optimality and all main equations for number of phase divisions and optimal number of levels for each partition are presented. These systems, although not optimal, may have asymptotic performance close to the optimum. Furthermore, the analysis and implementation can be simpler than those of optimal systems. PUPPQ has implementation complexity between optimal nonuniform polar quantization (NPQ) and uniform product polar quantization (UPPQ). The gain of PUPPQ over optimum uniform product polar quantization is also obtained (2 dB for the rate of 8 bits/sample).
\end{abstract}

Keywords: - asymptotical analysis, piecewise uniform product polar quantization, optimal distortion.

\section{INTRODUCTION}

Quantization is the heart of analog-to-digital conversion. Quantizers play an important role in the theory and practice of modern-day signal processing. Extensive results have been developed on scalar quantization but much more on vector quantization. The simplest vector quantization is polar quantization. Several studies have considered the design of suboptimal polar vector quantizers. These schemes should have provided better performances than those of the rectangularcoordinate-based quantizers, however with simpler implementation than optimal scalar quantizers. Polar quantization techniques as well as their applications in areas such as computer holography, discrete Fourier transform encoding, image processing and communications have been studied extensively in the literature. The solution of polar quantization problem is determination of decision and reproduction levels for every magnitude and phase value such that the distortion (MSE) is minimized. The resulting optimal quantizer is nonuniform. A special class of NPQ is a PUPPQ. Polar quantization consists of separate magnitude and phase quantizations, in $N$ levels, so that rectangular coordinates of the source $(x, y)$ are transformed into the polar coordinates in form: $r=\left(x^{2}+y^{2}\right)^{1 / 2}, \phi=\tan ^{-}$ ${ }^{1}(y / x)$ where $r$ represents magnitude and $\phi$ is phase. In previous works that involve polar quantization [1-
3 ], uniform product polar quantization (UPPQ) was considered $(N=M \times L)$. Uniform product polar quantization was optimized numerically in [1] and analytically in [2]. However, the analysis in [2] assumed a fixed support region. In paper [3] UPPQ was optimized asymptotically by uniform scalar quantization support region.

One of the most important results in polar quantization is due to Swaszek and $\mathrm{Ku}$ who derived the asymptotically optimal nonuniform polar quantization [4] (compressor-expander pair is needed). However, they didn't consider the problem of finding the optimal maximum amplitude, socalled, support region. The support region for scalar quantizers has been found in [5-6] by minimization of the total distortion $D$, which is a combination of granular $\left(D_{g}\right)$ and overload $\left(D_{o}\right)$ distortion, $D=D_{g}+D_{o}$. In paper [7] only granular distortion was examined and although, arrangement of points $N_{i}$ in L partitions was defined, cells type and their arrangement within partitions wasn't considered. Paper [8] is an annex of paper [7], but the imperfection of this paper lies in using cubic cells for partitions and subpartitions. Due to this fact, optimal arrangement of points in a partition can't be found. The importance of using the optimal density of points (using rectangular cells) for product quantization and Gaussian source is considered in $[9,10]$. The goal of this paper is to solve 
quantization problem in a case of PUPPQ and to find corresponding support region. It is performed by analytical optimization of the granular distortion and numerical optimization of the total distortion. If the distortion is measured by squared error, D becomes the mean squared error (MSE). Distortion mean-squared error (MSE i.e. quantization noise) is used as the criterion for optimization. The implementation is simple, and no compressorexpander pair is needed. We improve the cell size and use more optimal cell division in each partition. PUPPQ consists of $\mathrm{L}$ uniform product polar quantizers. More precisely, our quantizer divides the input plane into $L$ partitions and every partition is further subdivided into $L_{i}(1 \leq i \leq L)$ subpartitions. I-th partition in signal plane is allowed to have $M_{i}$ $(1 \leq i \leq L)$ cells in the phase quantizer. We perform two-steps optimization: 1) distortion optimization $\left(D_{i}\right)$ in every partition under the constraint $L_{i} x M_{i}=N_{i}$ and 2) optimization of the total granular distortion $D_{g}=\sum_{i=1}^{L} D_{i}$ which achieves the optimal number of points $N_{i}$ on each partition under the constraint $\sum_{i=1}^{L} N_{i}=N$. We also give an example of the quantizer construction for a Gaussian source. This case is of importance because using Gaussian quantizer on an arbitrary source we can take advantage of the central limit theorem and the known structure of an optimal scalar quantizer for a Gaussian random variable to code a general process by first filtering it to produce an approximately Gaussian density, scalar-quantizing the result, and then inverse-filtering to recover the original . Various processing techniques, when applied to non-Gaussian sources with memory, produce sequences, which are "approximately" independent, and Gaussian.

The paper is organized as follows. In Section II we give simple general conditions for optimal product polar uniform quantization and prove its optimality. In Section III we apply the solution from section II on PUPPQ, give an equation for optimal number of points per partition and we derive $D_{\mathrm{g}}$ in a closed form. In Section IV we give simple construction procedure and present the Gaussian source example. Section V gives some conclusions.

\section{OPTIMAL UNIFORM PRODUCT POLAR QUANTIZATION}

We assume data to be encoded as a pair of zeromean , unit-variance, independent identically distributed Gaussian random variables. The data vector $(\mathrm{x}, \mathrm{y})$ has a joint density given by

$$
f(x, y)=\frac{1}{2 \pi} \cdot e^{\frac{-\left(x^{2}+y^{2}\right)}{2}} ;-\infty \leq x, y \leq \infty
$$

Uniform product polar quantization to $N$ levels consists of transforming the rectangular coordinate representation of the source $(\mathrm{x}, \mathrm{y})$ to the polar coordinates of magnitude $\mathrm{r}$ and phase $\phi$ through: $r=\left(x^{2}+y^{2}\right)^{1 / 2}, \phi=\tan ^{-1}(y / x)$ and quantizing $r$ and $\phi$ separately by uniform scalar quantizers with $\mathrm{L}$ and M levels $(L x M=N)$, respectively. With this scheme the bivariate quantization pattern is a partitioning of the plane into L concentric rings around the origin, each divided angularly into $\mathrm{M}$ pieces. This is natural for circularly symmetric densities such as independent identically distributed Gaussian random variables. Transforming to polar coordinates, the phase is uniformly distributed on $[0,2 \pi)$ and the magnitude is distributed on $[0, \infty)$ with density function $f(r)=r e^{\frac{-r^{2}}{2 \sigma^{2}}}$. Note that magnitude and phase are independent random variables. The transformed joint density function for the Gaussian source is

$$
f(r, \phi)=\frac{1}{2 \pi} \cdot r e^{\frac{-r^{2}}{2}}=\frac{f(r)}{2 \pi}
$$

Lets consider uniform product polar quantizer of $L$ magnitude levels and $M$ phase reproduction levels on a magnitude reproduction level $m_{\mathrm{i}}, 1 \leq i \leq L$. Magnitude decision levels and reproduction levels are given as (see Fig.1):

$r_{i}=(i-1) \Delta, 1 \leq i \leq L+1 ; m_{i}=(i-1 / 2) \Delta, 1 \leq i \leq L$ where $\Delta=r_{\max } / L$.

Further we divide a partition of each magnitude ring into $M$ phase subpartitions. Let $\phi_{i, j}$ and $\phi_{i, j+1}$ be two phase decision levels, and let $\psi_{i, j}$ be $j$-th phase reproduction level for the $i$-th magnitude ring, $1 \leq j \leq$ $M$. It follows that: $\phi_{i, j}=(j-1) 2 \pi / M$ and $\psi_{i, j}=(2 j-1) \pi / M$. We can define a quantizer consisting of a set of granular cells and a set of overload cells 

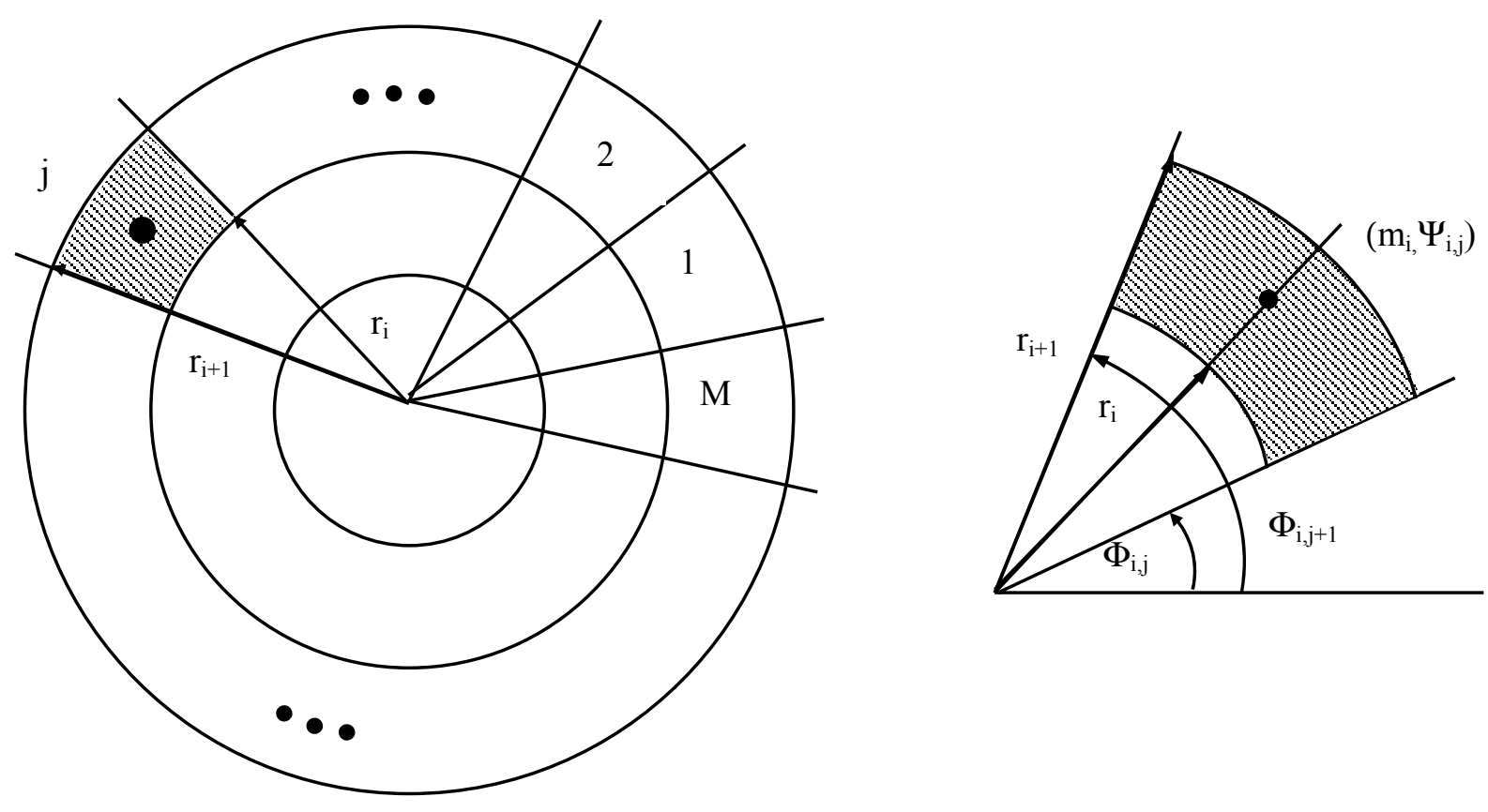

Fig.1 - Magnitude and phase division in UPPQ and $j$-th cell on $i$-th level preview

$S_{g}=\left\{S_{i, j}=\left(\left[r_{i}, r_{i+1}\right) ;\left\lfloor\phi_{i, j}, \phi_{i, j+1}\right)\right) ; 1 \leq i \leq L, 1 \leq j \leq M\right\}$

$d(\mathbf{r}, \mathbf{c})$ that quantifies cost or distortion resulting

$S_{o}=\left\{S_{L, j}^{o}=\left(\left[r_{\max }, \infty\right) ;\left[\phi_{L, j}, \phi_{L, j+1}\right)\right) ; 1 \leq j \leq M\right\}$

together with a set of reproduction vectors from reproducing $\mathbf{r}$ as $\mathbf{c}$ and to consider the average distortion as a measure of the quality of a system,

$$
C=\left\{\mathbf{c}_{i, j}=\left(m_{i}, \psi_{i, j}\right) ; 1 \leq i \leq L, 1 \leq j \leq M\right\}
$$
with smaller average distortion meaning higher quality. The most common distortion measure is the squared error

$$
d(\mathbf{r}, \mathbf{c})=|\mathbf{r}-\mathbf{c}|^{2}=r^{2}+m^{2}-2 r m \cos (\phi-\psi) .
$$

(see Fig.1). All of overload (granular) cells are called the overload region (granular region).

The quality of a quantizer can be measured by the goodness of the resulting reproduction in comparison to the original. One way of accomplishing this is to define a distortion measure

In practice, the average will be a sample average when the quantizer is applied to a data sequence. The theory views the data as sharing a common probability density function $f(r, \phi)$ corresponding to the average distortion per dimension becomes an expectation

$$
D=D_{g}+D_{o}=\frac{1}{2}\left(\sum_{i=1}^{L} \sum_{j=1}^{M} \int_{S_{i, j}} d\left(\mathbf{r}, \mathbf{c}_{i, j}\right) f(r, \phi) d r d \phi+\sum_{j=1}^{M} \int_{S_{L, j}^{o}} d\left(\mathbf{r}, \mathbf{c}_{L, j}\right) f(r, \phi) d r d \phi\right) .
$$

If the distortion is measured by squared error, D becomes the mean squared error (MSE).
In order to minimize the distortion we proceed as follows. $D$ can be written as: 


$$
\begin{aligned}
D=D_{g}+D_{o} & =\frac{1}{2} \sum_{i=1}^{L} \sum_{j=1}^{M} \int_{\phi_{i, j}}^{\phi_{i, j+1}} \int_{r_{i}}^{r_{i+1}}\left[r^{2}+m_{i}^{2}-2 r m_{i} \cos \left(\phi-\psi_{i, j}\right)\right] \frac{f(r)}{2 \pi} d r d \phi \\
& +\frac{1}{2} \sum_{j=1}^{M} \int_{\phi_{L, j}}^{\phi_{L, j+1}} \int_{r_{\max }}^{\infty}\left[r^{2}+m_{L}^{2}-2 r m_{L} \cos \left(\phi-\psi_{L, j}\right)\right] \frac{f(r)}{2 \pi} d r d \phi
\end{aligned}
$$

After integration over $\phi$ and reordering, $D$ becomes

$$
D=\frac{1}{2}\left(\sum_{i=1}^{L} \int_{r_{i}}^{r_{i+1}}\left[r^{2}+m_{i}^{2}-2 r m_{i} \sin c\left(\frac{\pi}{P_{i}}\right)\right] f(r) d r+\int_{r_{\max }}^{\infty}\left[r^{2}+m_{L}^{2}-2 r m_{L} \sin c\left(\frac{\pi}{M}\right)\right] f(r) d r\right)
$$

Wherein $\operatorname{sinc}(x)=\sin (x) / x$ and we use : $\frac{\sin (x)}{x} \approx 1-\frac{1}{6} x^{2}$ so that

$$
D \approx \frac{1}{2}\left(\sum_{i=1}^{L} \int_{r_{i}}^{r_{i+1}}\left[\left(r-m_{i}\right)^{2}+\frac{r m_{i}}{3} \frac{\pi^{2}}{M^{2}}\right] f(r) d r+\int_{r_{\max }}^{\infty}\left[\left(r-m_{L}\right)^{2} f(r) d r+\frac{\pi^{2} m_{L}}{3 M^{2}} \int_{r_{\max }}^{\infty} r f(r) d r\right)\right.
$$

After the approximation

$\int_{r_{i}}^{r_{i+1}} r f(r) d r \approx m_{i} f\left(m_{i}\right) \Delta$, it follows

$$
\begin{aligned}
D_{g} & \approx \frac{1}{2}\left(\frac{\Delta^{2}}{12} \sum_{i=1}^{L} f\left(m_{i}\right) \Delta+\frac{\pi^{2}}{3 M^{2}} \sum_{i=1}^{L} m_{i}^{2} f\left(m_{i}\right) \Delta\right) \approx \frac{1}{2}\left(\frac{\Delta^{2}}{12} \int_{0}^{r_{\max }} f(r) d r+\frac{\pi^{2}}{3 M^{2}} \int_{0}^{r_{\max }} r^{2} f(r) d r\right)= \\
& =\frac{1}{2}\left(\frac{\Delta^{2}}{12} P+\frac{\pi^{2}}{3 M^{2}} I\right)
\end{aligned}
$$

Approximating sums by integrals $(\Delta \approx d r)$, granular and overload distortions become

$$
\begin{aligned}
& D_{g}=\frac{1}{2}\left(\frac{\Delta^{2}}{12} P+\frac{\pi^{2}}{3 M^{2}} I\right) \\
& D_{o}=\frac{1}{2}\left(\int_{r_{\max }}^{\infty}\left[\left(r-m_{L}\right)^{2} f(r) d r+\frac{\pi^{2} m_{L}}{3 M^{2}} \int_{r_{\max }}^{\infty} r f(r) d r\right)\right.
\end{aligned}
$$

For large $r_{\max }, P \approx 1, I \approx 2$ granular distortion becomes equal to the well-known distortion [1-3] $D_{g}=\frac{1}{2}\left(\frac{\Delta^{2}}{12}+\frac{2 \pi^{2}}{3 M^{2}}\right)$. In order to optimize granular distortion, we substitute expressions $M=N / L, \Delta=r_{\max } / L=\Delta_{0} / L, \quad\left(\Delta_{0}=r_{L+1}-r_{1}\right)$ in (7)

$D_{g}=\frac{1}{2}\left(\frac{1}{12} \frac{\Delta_{0}^{2}}{L^{2}} P+\frac{\pi^{2} L^{2} I}{3 N^{2}}\right)$

After solving $\frac{\partial D_{i}}{\partial L}=0$ we obtain

$$
\begin{aligned}
& L_{o p t}=\sqrt[4]{\frac{\Delta_{0}^{2} P N^{2}}{4 \pi^{2} I}} \\
& M_{o p t}=N / L_{o p t}=\sqrt[4]{\frac{4 \pi^{2} I N^{2}}{\Delta_{0}^{2} P}} \\
& \text { that gives } \\
& D_{g}=\frac{1}{6} \frac{\Delta_{0} \pi \sqrt{P I}}{N} \\
& \text { the minimum distortion } D_{g}^{\text {opt }} \text { because of } \\
& \frac{\partial^{2} D_{g}}{\partial L^{2}}>0 \text {. }
\end{aligned}
$$

\section{PIECEWISE UNIFORM PRODUCT POLAR QUANTIZATION}

The most important results in polar quantization are ascribed to Swaszek and $\mathrm{Ku}$ who derived the asymptotically optimal unrestricted polar quantization (NPQ) [4]. The nonlinear compressor 
characteristic is used in paper [4]. Although the smooth and differentiable compressor characteristic is convenient for mathematical manipulations, there are problems of accurately implementing analog nonlinearities [11]. Today's technology allows uniform quantizers or piecewise linear compressor characteristics implementation.

A piecewise uniform product polar quantizer consists of $\mathrm{L}$ different uniform product polar quantizers. Different quantizers, however, may have different step-sizes. In this paper, we give the simplest piecewise uniform quantization and show that it has approximately same performances as NPQ but it's much simpler for application.

Let consider PUPPQ of $L$ partitions, each partition containing $L_{i}$ subpartitions. In order to minimize the total distortion we proceed as follows: magnitude partition decision levels and reproduction subpartition levels are given as (see Fig.2)
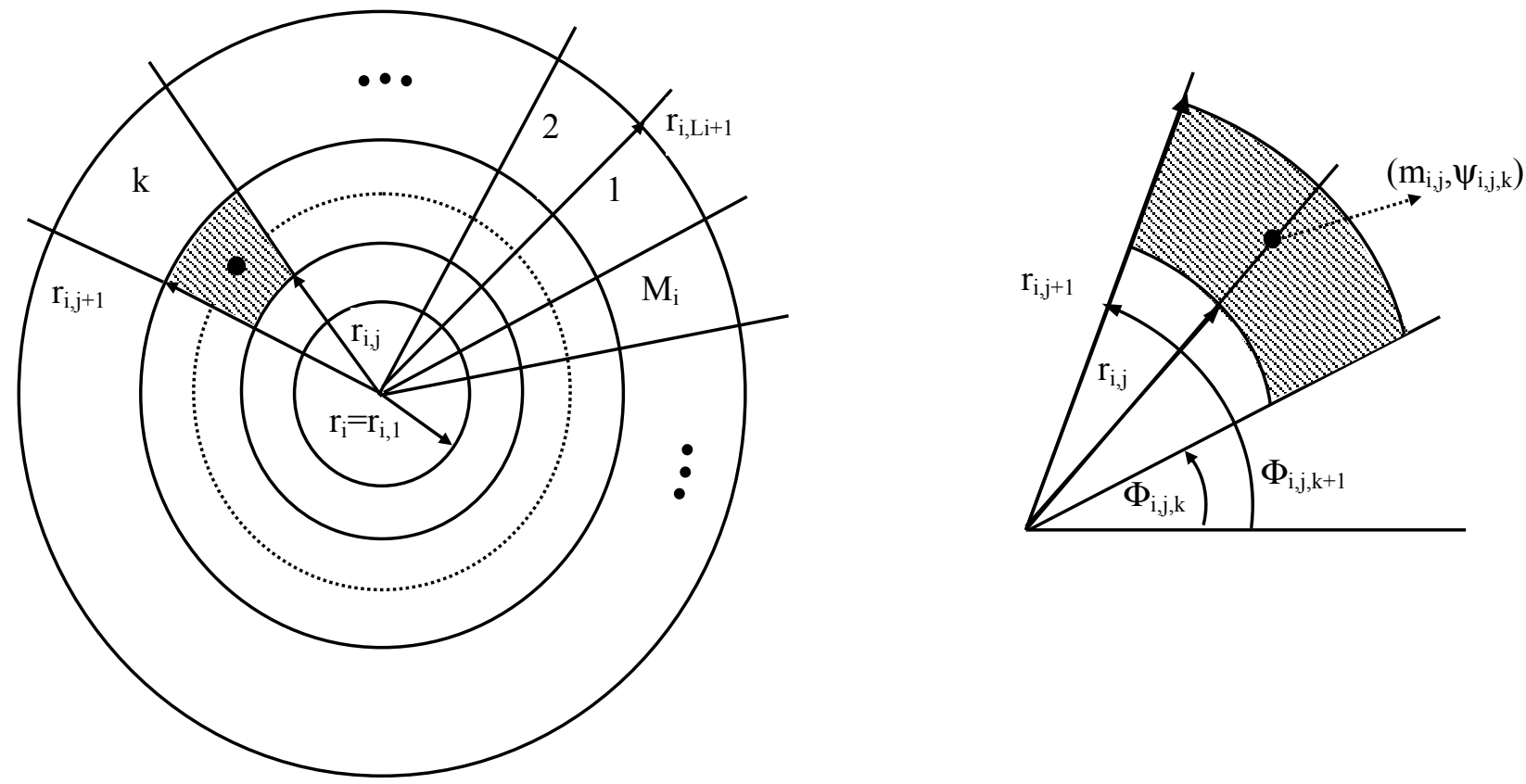

Fig.2 - I-th partition of PUPPQ and $k$-th cell on $j$-th level

$$
\begin{aligned}
& r_{i}=(i-1) \Delta ; 1 \leq i \leq L ; \quad r_{L+1}=r_{\max } \\
& r_{i, j}=r_{i}+(j-1) \Delta / L_{i} ; 1 \leq i \leq L, \quad 1 \leq j \leq L_{i}+1 ; r_{L, L_{L+1}}=r_{L+1}=r_{\max } \\
& m_{i, j}=r_{i}+(j-1 / 2) \Delta / L_{i} ; \quad 1 \leq i \leq L, \quad 1 \leq j \leq L_{i}
\end{aligned}
$$

where $\Delta=r_{\max } / L$. Let $\phi_{i, j, k}$ be phase decision level, and let $\psi_{i, j, k}$ be $k$-th phase reproduction level for the $i$-th partition and $j$-th subpartition. Then $\phi_{i, j, k}=(k-1) 2 \pi / M_{i} ; 1 \leq k \leq M_{i}+1$ and $\psi_{i, j, k}=(2 k-1) \pi / M_{i} ; 1 \leq k \leq M_{i}$.

The minimization of the function $D_{g}(\mathbf{N}, \mathbf{M}, \mathbf{L})$ (vectors $\left.(\mathbf{N}, \mathbf{L}, \mathbf{M})=\left(N_{i}, L_{i}, M_{i}\right)_{1 \leq i \leq L}\right)$ for a fixed number of magnitude levels $L$ constrained by the total number of reproduction points $N$ is formulated in this way: minimize $D_{g}(\mathbf{N}, \mathbf{M}, \mathbf{L})$ under the constraints $\sum_{i=1}^{L} L_{i} M_{i}=\sum_{i=1}^{L} N_{i}=N$.
UPPQ with $L=L_{i}, \quad M=M_{i}, \quad N_{i}=M_{i} L_{i}$, $\Delta_{0}=\Delta, P_{i}=\int_{r_{i}}^{r_{i+1}} f(r) d r$ and $I_{i}=\int_{r_{i}}^{r_{i+1}} r^{2} f(r) d r$ is applied on each partition. The $i$-th partition distortion is $D_{i}=\frac{1}{2}\left(\frac{1}{12} \frac{\Delta^{2}}{L_{i}^{2}} P_{i}+\frac{\pi^{2} I_{i}}{3 M_{i}^{2}}\right)$.

The granular distortion is a sum of partition distortions $D_{i}$ :

$$
D_{g}=\sum_{i=1}^{L} D_{i}=\sum_{i=1}^{L} \frac{1}{2}\left(\frac{1}{12} \frac{\Delta^{2}}{L_{i}^{2}} P_{i}+\frac{\pi^{2} I_{i}}{3 M_{i}^{2}}\right)
$$


In a previous section, we pointed out that optimal values may be found by following equations:

$$
\begin{aligned}
L_{\text {iopt }} & =\sqrt[4]{\frac{\Delta^{2} P_{i} N_{i}^{2}}{4 \pi^{2} I_{i}}} ; \quad M_{i o p t}=\sqrt[4]{\frac{4 \pi^{2} I_{i} N_{i}^{2}}{\Delta^{2} P_{i}}} \\
D_{\text {iopt }} & =\frac{1}{6} \frac{\Delta \pi \sqrt{P_{i} I_{i}}}{N_{i}}
\end{aligned}
$$

The overload distortion is:

$$
D_{o}=\frac{1}{2}\left(\int_{r_{\max }}^{\infty}\left[\left(r-m_{L . L_{L}}\right)^{2} f(r) d r+\frac{\pi^{2} m_{L . L_{L}}}{3 M_{L}^{2}} \int_{r_{\max }}^{\infty} r f(r) d r\right)\right.
$$

Substituting (14) in equation for granular distortion we obtain

$$
D_{g}=\frac{1}{6} \Delta \pi \sum_{i=1}^{L} \frac{\sqrt{P_{i} I_{i}}}{N_{i}}
$$

The optimization of granular distortion (16) can be formulated in this way: we use equation $J=D_{g}+\lambda \sum_{j=1}^{L_{i}} N_{i} \quad$ where $\lambda \quad$ represents Lagrangian multiplier and after solving $\frac{\partial J}{\partial N_{i}}=0$ under constraint $\sum_{j=1}^{L} N_{i}=N$ we obtain

$$
N_{i}=\frac{N \sqrt[4]{P_{i} I_{i}}}{\sum_{j=1}^{L} \sqrt[4]{P_{j} I_{j}}}
$$

These are optimal values for $N_{i}$ because $D_{g}\left(N_{1}, \ldots, N_{L}\right)$ is convex function and constraint $\sum_{j=1}^{L} N_{i}=N$ forms convex set (Hessian matrix are positive semi definite).

Finally, granular distortion becomes

$$
D_{g}=\frac{1}{6} \frac{\Delta \pi}{N}\left(\sum_{i=1}^{L} \sqrt[4]{P_{i} I_{i}}\right)^{2}
$$

The exact optimal value for $r_{\max }$ is obtained repeating our optimization method for different $r_{\max }$ and choosing the values for which $D=D_{g}+D_{o}$ is minimal.

\section{DESIGN OF OPTIMAL PIECEWISE UNIFORM PRODUCT POLAR QUANTIZER}

In this section we will give step-by-step procedure for obtaining PUPPQ. For a fixed set number $\mathrm{N}$ we determine $\left(N_{i} L_{i} M_{i}\right)$ for a fixed $r_{\max }, \Delta=r_{\max } / L$

Step 1)

$$
N_{i}=\frac{N \sqrt[4]{P_{i} I_{i}}}{\sum_{j=1}^{L} \sqrt[4]{P_{j} I_{j}}}
$$

Step 2)

$$
\begin{gathered}
L_{\text {iopt }}=\sqrt[4]{\frac{\Delta^{2} P_{i} N_{i}^{2}}{4 \pi^{2} I_{i}}} ; \\
M_{\text {iopt }}=\sqrt[4]{\frac{4 \pi^{2} I_{i} N_{i}^{2}}{\Delta^{2} P_{i}}}
\end{gathered}
$$

where $P_{i}=\int_{r_{i}}^{r_{i+1}} f(r) d r$ and $I_{i}=\int_{r_{i}}^{r_{i+1}} r^{2} f(r) d r$.

Step 3) The exact optimal value for $r_{\max }$ is obtained by repeating our optimization method for different $r_{\max }$ and choosing the values for which $D=D_{g}+D_{o}$ is minimal. The optimum numbers $\left(N_{i}, L_{i}, M_{i}\right)_{1 \leq i \leq L}$ in each partition were found by performing again the evaluation taking the nearest integer values. If a specific value of $N$ is desired, the $\left(N_{i}, L_{i}, M_{i}\right)_{1 \leq i \leq L}$ values can be adjusted (rounded up or down) to sum to $N$ exactly or nearest $N$, in order to minimize $D_{g}$.

Step 4) The decision levels and reproduction levels are:

$$
\begin{gathered}
r_{i, j}=r_{i}+(j-1) \Delta / L_{i} ; 1 \leq i \leq L, \quad 1 \leq j \leq L_{i}+1 ; r_{L, L_{L+1}}=r_{L+1}=r_{\max } \\
m_{i, j}=r_{i}+(j-1 / 2) \Delta / L_{i} ; \quad 1 \leq i \leq L, \quad 1 \leq j \leq L_{i} \\
\phi_{i, j, k}=(k-1) 2 \pi / M_{i} ; 1 \leq k \leq M_{i}+1 \\
\psi_{i, j, k}=(2 k-1) \pi / M_{i} ; 1 \leq k \leq M_{i} .
\end{gathered}
$$

As an illustration of the PUPPQ performance, we show the signal-to-quantization noise ratio $S N R=10 \log (1 / D)$ as a function of the number of bits per sample $R\left(R=\log _{2} N\right)$.

In order to see advantages of PUPPQ we performed numerical calculations of total distortion for $\mathrm{L}=1,4,8$ and rates $\mathrm{R}=(4-8)$ bits/sample. PUPPQ gain compared to UPPQ is up to $2 \mathrm{~dB}(\mathrm{~L}=8)$ (Fig.3). 
By exceeding L, better performances may be achieved but complexity becomes greater. Theoretical bound for the optimal NPQ is $D=2 \pi /(3 N)=2.09 N^{-1}$ which is for only $0.17 \mathrm{~dB}$ worse than for the case of optimal two-dimensional vector quantization $D=10 \pi /(9 \sqrt{3} N)=2.016 N^{-1}$ $[4,9]$. The available design algorithms have very slow convergence unless the rate-dimension product is small [4, 9], and implementation of the optimum vector quantizer is a computationally burdensome full search procedure. The comparison of PUPPQ $(\mathrm{L}=8)$ with theoretical distortion is shown on Fig.4. Theoretical bound for polar quantization is possible to achieve for large $\mathrm{L}(L \geq 50)$ which is impractical because of negligible gain and increased complexity in regard to $\mathrm{L}=8$.

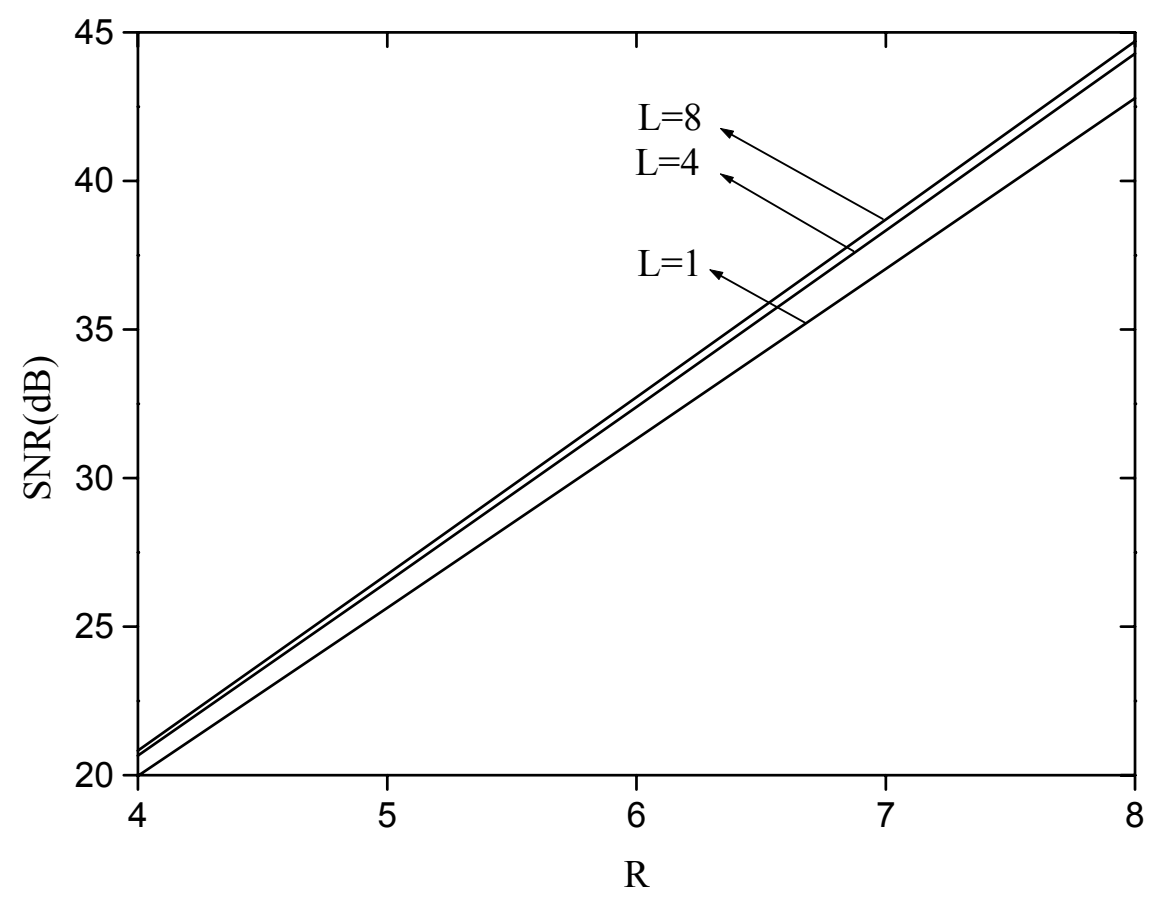

Fig. 3 - PUPPQ performances (SNR) versus rate for different numbers of partitions

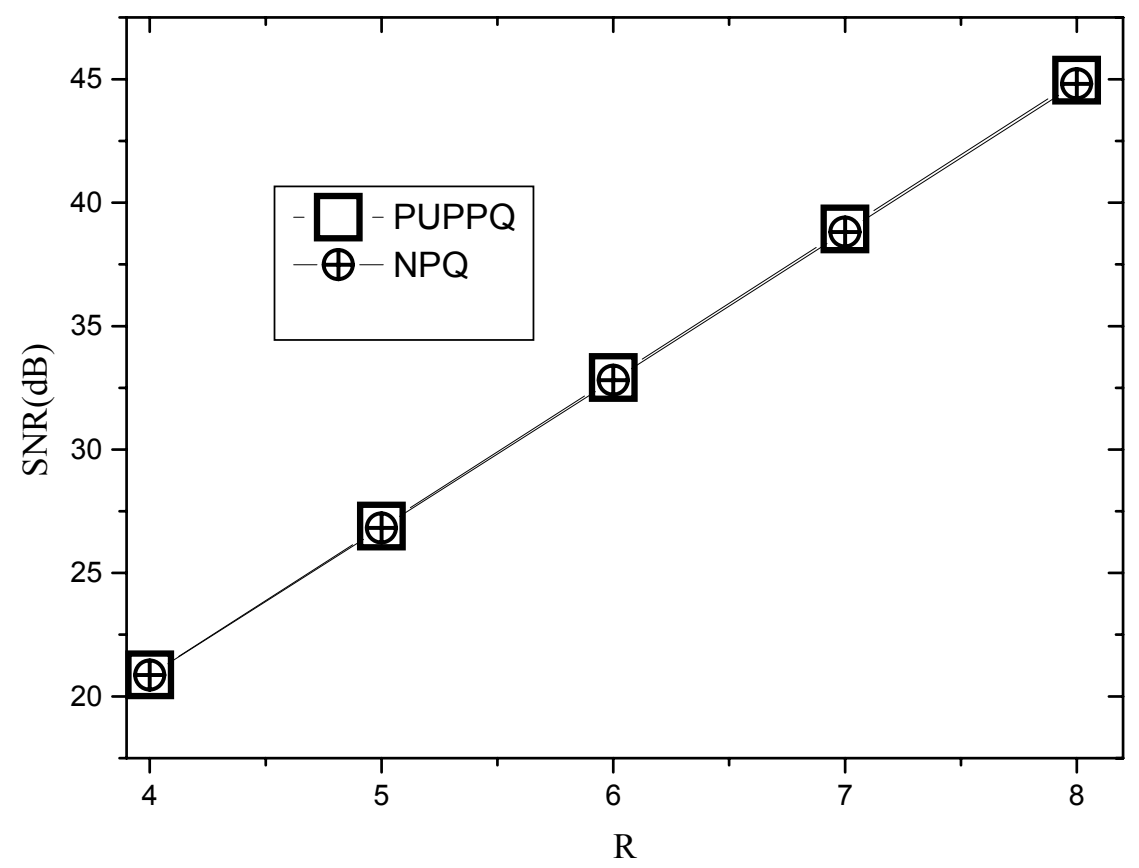

Fig.4. PUPPQ performances (SNR) versus rate for $L=8$ compared to theoretical NPQ bound [4] 
Application of NPQ requires nonlinear compressor, expander and lookup table of number phase divisions. For $\mathrm{R}=8$ lookup table has $L=\sqrt{N / 2}=181$ elements, while PUPPQ for $\mathrm{L}=8$ requires: lookup table of 17 memory elements $\left(\left(L_{i}, M_{i}\right), r_{\max }\right)$ and no nonlinear compressor.

It is obvious that our results are very close to the best. However, if we compare PUPPQ results for $\mathrm{L}=14$ with those given in paper [4] we have $\mathrm{D}^{\mathrm{PUPPQ}}=2.149 \mathrm{~N}^{=1}$ while $\mathrm{D}^{[4]}=2.153 \quad \mathrm{~N}^{=1}$, which means that for greater L our results would be better than best known. The reason of obtaining better results lies in a fact that the method we proposed defines optimal support region.

For $\mathrm{L}=8$ and rates $\mathrm{R}=(4,6,8)$ optimal integer values of $\left(\mathrm{L}_{\mathrm{i}}, \mathrm{M}_{\mathrm{i}}\right)$ are given in Table 1 .

Table 1.

\begin{tabular}{|c|c|c|c|c|c|c|}
\hline$=8$ & \multicolumn{2}{|c|}{$\begin{array}{c}\mathrm{R}=4 \\
\mathrm{r}_{\max }=3.666\end{array}$} & \multicolumn{2}{|c|}{$\begin{array}{c}\mathrm{R}=6 \\
\mathrm{r}_{\max }=4.465\end{array}$} & \multicolumn{2}{|c|}{$\begin{array}{c}\mathrm{R}=8, \\
\mathrm{r}_{\max }=5.054\end{array}$} \\
\hline $\mathrm{i}$ & L & $\mathrm{M}$ & L & M & $\mathrm{L}$ & $\mathrm{M}$ \\
\hline 1 & 2 & 6 & 9 & 1 & $0^{4}$ & $\begin{array}{c}1 \\
76\end{array}$ \\
\hline 2 & 2 & $5^{1}$ & 9 & ${ }^{9}$ & $9^{3}$ & $83^{3}$ \\
\hline 3 & 2 & $1^{2}$ & 8 & $22^{1}$ & $3^{3}$ & $08^{5}$ \\
\hline 4 & 1 & $7^{4}$ & 6 & $\begin{array}{r}1 \\
41\end{array}$ & $5^{2}$ & $29^{5}$ \\
\hline 5 & 1 & $4^{4}$ & 5 & $\begin{array}{c}1 \\
15\end{array}$ & $7^{1}$ & $\begin{array}{c}4 \\
68\end{array}$ \\
\hline 6 & 1 & $6^{3}$ & 3 & $06^{1}$ & 1 & $42^{3}$ \\
\hline 7 & 1 & $6^{2}$ & 2 & $2^{7}$ & 6 & $\begin{array}{l}2 \\
37\end{array}$ \\
\hline 8 & 1 & $8^{1}$ & 1 & $5^{5}$ & 3 & $\begin{array}{c}1 \\
45^{1}\end{array}$ \\
\hline
\end{tabular}

If we compare our results for $\mathrm{R}=4(\mathrm{~L}=8)$ to those from paper [8] gain is $0.375 \mathrm{~dB}$. Greater gain can be achieved for greater $\mathrm{L}$ and $\mathrm{R}$.

Application of Polar Quantization:

Short-time pdf of speech segments are described by Gaussian pdf [13]. This paper addresses potential improvements achievable by means of joint quantization of two consecutive samples (x,y), referred to as a two-dimensional quantization (2-D quantization), over the scalar quantization. Transform coding scheme known as spectral phase coding (SPC), is a robust technique for coding a nonstationary or large dynamic range discrete-time series into digital form. SPC is, essentially, a polar format representation of a random phase time series discrete Fourier transform (DFT). SPC utilizes DFT and a two-dimensional quantizer to obtain its robust characteristics.

The design of optimal uniform polar quantization method is presented in image processing applying it on complex reflectivity function in SAR systems [14]. Application of optimal polar quantization in SAR systems will provide better results for about $0.5 \mathrm{~dB}$. Optimal polar quantization may be applied to Adaptive Differential Pulse Code Modulation (ADPCM). In ADPCM systems it utilizes uniform scalar quantization [15]. Optimal uniform scalar quantization for $\mathrm{R}=4$ (bit/sample) has $\mathrm{SNR}=19.38 \mathrm{~dB}$ [16] until optimal polar quantization has $\mathrm{SNR}=20.76$. Optimal PUPPQ may achieve gain of about $1.38 \mathrm{~dB}$ in regard to Optimal Scalar Quantization.

\section{CONCLUSION}

In this paper, simple general conditions for optimal product polar uniform quantization that prove its optimality are presented. The simple asymptotical analysis is given for piecewise uniform product polar quantizer with an algorithm for its construction, for any number of points $\mathrm{N}$ (FixedRate). The algorithm is demonstrated on the Gaussian source example. For the fixed number of partitions, we give equations for optimal number of levels $L_{i}$ and optimal number of phase divisions $M_{i}$. We also give optimal number of points $N_{i}$ for each partition, while optimal support region is obtained numerically. For $\mathrm{L}=8$, PUPPQ performances are very close to NPQ theoretical bound, while the gain is up to $2 \mathrm{~dB}$ (for $\mathrm{L}=8$ ) in regard to optimal product polar quantization. The PUPPQ method achieves nearly theoretical NPQ performances with less complexity.

Polar Quantization has a great application nowadays and we predict that it would have greater application in future.

\section{REFERENCES}

[1] W. A. Pearlman, "Polar quantization of complex Gaussian random variable, " IEEE Trans. Commun., vol. COM-27, pp. 892-899, June 1979.

[2] P. F. Swaszek, "Uniform spherical coordinate quantization of spherically symmetric sources", IEEE Trans. Commun., vol. COM-33, pp. 518-521, June 1985.

[3] P. W. Moo, D. L. Neuhoff, "Uniform Polar Quantization Revisited," In Proc. IEEE Int. Symp. Information Theory ISIT'98, pp. 100, Cambridge, USA, August 1998.

[4] P. F. Swaszek, T. W. Ku, "Asymptotic Performance of Unrestricted Polar Quantizer", 
IEEE Transactions on Information Theory, vol. 32, pp. 330-333, 1986.

[5] D. Hui, D. L. Neuhoff, "Asymmptotic Analysis of Optimal Fixed-Rate Uniform Scalar Quantization," IEEE Transaction on Information Theory, vol.47, pp. 957-977, March 2001.

[6] S. Na, D. L. Neuhoff, "On the Support of MSE-Optimal, Fixed-Rate Scalar Quantizers" IEEE Transaction on Information Theory, vol.47, pp. 2972-2982, November 2001.

[7] F. Kuhlmann and J. A. Bucklew, "Piecewise Uniform Vector Quantizers", IEEE Transactions on Information Theory, vol. 34, pp. 1259-1263, 1988.

[8] P. F. Swaszek, "Unrestricted Multistage Vector Quantizers", IEEE Transactions on Information Theory, vol. 38, pp. 1169-1174, 1992.

[9] R. M. Gray and D. L. Neuhoff, "Quantization", IEEE Transactions on Information Theory, vol. 44, no. 6, pp. 2325-2384, October 1998.

[10] S. Na D. L. Neuhoff, "Bennett's Integral for Vector Quantizers," IEEE Transaction on Information Theory, vol.41, pp. 886-900, July 1995.

[11] A. Gersho and R. M. Gray, "Vector Quantization and signal Compression", Kluwer Academ.Pub(1992).

[12] A. Gersho, "Asymptotically Optimal Block Quantization" IEEE Transaction on Information Theory, vol.25 pp. 373-380, July 1979.

[13] N. S. Jayant and P. Noll, "DIGITAL CODING OF WAVEFORMS Principles and Applications to Speech and Video", Prentice-Hall, New Jersey (1984).

[14] Z. H. Peric, J. D. Jovkovic, "Application of the Optimal Uniform Polar Quantization on Complex Reflectivity Function" Advances in Electrical and Computer Engineering, vol.2 (9) $\mathrm{Nr}$ 1(17), pp. 80-85,2002.

[15] D. Minoli, "Voice Over MPLS Planning and Designing Networks", McGraw-Hill.Pub(2002).

[16] D. G. Jeong, J. Gibson" Uniform and Piecewise Uniform Lattice Vector Quantization for Memoryless Gaussian and Laplacian Sources", IEEE Trans., 1993, IT-39(3), pp. 786-804.

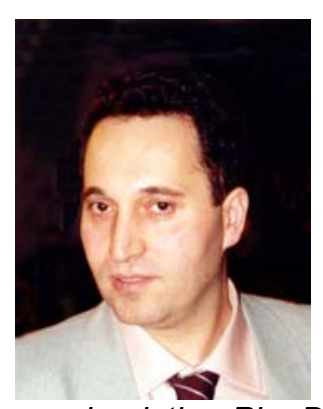

Zoran $\boldsymbol{H}$. Peric was born in Nis, Yugoslavia, in 1964. He received the $B$. Sc. degree in electronics and telecommunications from the Faculty of Electronic Engineering, Nis, Serbia, Yugoslavia, in 1989, and $M$. Sc. degree in telecommunications from the University of Nis, in 1994. He received the $P h$. $D$. degree from the University of Nis, also, in 1999.
He is currently Professor at the Department of Telecommunications, University of Nis, Serbia\&Montenegro. His current research interests include the information theory, source and channel coding and signal processing. He is particulary working on scalar and vector quantization techniques in image compression. He was author and coauthor in over 60 papers in digital communications. Dr Peric has been a Reviewer for IEEE Transactions on Information Theory.

Daniela M. Milovic was born in Nis, Yugoslavia, in 1970. She received the B. Sc. Degree in electronics and telecommunications from the Faculty of Electronic Engineering, Nis, Serbia, Yugoslavia, in 1995, and $M$. Sc. Degree in telecommunications from the Univeristy of Nis, in 1999. She received Ph. D. degree from the University of Nis in 2003.

She is currently teaching and research assistant with the Department of Telecommunications, University of Nis, Serbia\&Montenegro. Her current interests include the information theory, source coding, polar quantization and nonlinear fiber optics. 